\title{
Impact of Nuclear Reactions on the Fate of Intermediate-mass Stars
}

\author{
Heiko Möller* \\ Technische Universität Darmstadt, Germany \\ E-mail: hmoeller@theorie.ikp.physik.tu-darmstadt.de
}

\section{Samuel Jones}

University of Victoria, Canada

E-mail: swjones@uvic.ca

Tobias Fischer

Uniwersytet Wroctawski, Poland

E-mail: fischer@ift.uni.wroc.pl

\section{Gabriel Martínez-Pinedo}

Technische Universität Darmstadt, Germany

E-mail: gabriel.martinez@physik.tu-darmstadt.de

Stars in the mass range of 8 to $12 \mathrm{M}_{\odot}$ (solar masses) represent the transition region between those that end their lives producing white dwarfs and those that undergo a core-collapse supernova and produce neutron stars.

The final phases of stellar evolution of these intermediate-mass stars are tightly connected to the behavior of nuclear reactions at high densities. Despite their importance, the stellar evolution of intermediate-mass stars has received little attention in the past. The pioneering work in this mass range was published by Nomoto in the 80's [1,2]. Numerical and computational difficulties had hindered progress in the past, however two recent stellar evolution studies $[3,4]$ revived the interest in intermediate-mass stars.

We explore nuclear processes that may be relevant for the modeling of these stars, starting from the neon burning stage. We show, that due to electron captures on ${ }^{20} \mathrm{Ne},{ }^{20} \mathrm{O}$ becomes abundant in the stellar core. This opens new reaction channels that have so far not been considered. These reactions modify the standard neon-burning that now proceeds by the reactions ${ }^{20} \mathrm{Ne}(\gamma, \alpha){ }^{16} \mathrm{O}$, followed by ${ }^{20} \mathrm{O}(\alpha, \gamma){ }^{24} \mathrm{Ne}$. Once the stellar core reaches a sufficiently high temperature, also the fusion reactions of neutron-rich oxygen isotopes, ${ }^{16} \mathrm{O}+{ }^{20} \mathrm{O} \rightarrow{ }^{36} \mathrm{~S}^{*}$ and ${ }^{20} \mathrm{O}+{ }^{20} \mathrm{O} \rightarrow{ }^{40} \mathrm{~S}^{*}$, may become important.

XIII Nuclei in the Cosmos,

7-11 July, 2014

Debrecen, Hungary

\footnotetext{
* Speaker.
} 


\section{Introduction}

Intermediate-mass stars from 8 to $12 \mathrm{M}_{\odot}$ are very abundant. According to ZAMS (zeroage main sequence) mass estimates for stars connected to supernova remnants observed in M31, intermediate-mass stars are even more abundant than the value of $42 \%$ which is suggested by the Salpeter IMF [5]. Nevertheless, there is a distinct lack of stellar evolution models in that mass range.

Looking at the nuclear physics involved, these objects are especially interesting because compared to massive stars (i.e. above $12 \mathrm{M}_{\odot}$ ), nuclear reactions operate at much higher densities. For example, neon burning operates at densities above $10^{9} \mathrm{~g} \mathrm{~cm}^{-3}$ whereas in massive stars the densities are considerably lower at around $10^{6} \mathrm{~g} \mathrm{~cm}^{-3}$. In addition to that, there is also a lack of sensitivity exploration concerning the nuclear physics input, which is crucial for the understanding of these stars. This concerns weak processes like the electron capture on nuclei (EC) but also processes like $(\alpha,\{\gamma, \mathrm{n}, \mathrm{p}\})$-reactions and fusion on the other side.

Concerning the use of intermediate-mass star progenitors in supernova simulations, such models are particularly interesting because unlike their massive counterparts, an explosion can be obtained in spherically symmetric models. This in return allows for a more detailed study of the involved nucleosynthesis processes and neutrino input physics.

\section{Nuclear physics input for stellar evolution models of intermediate-mass stars}

Intermediate-mass stars denote the transition region of stars that can become either O-Ne white dwarfs or undergo a gravitational collapse or even explode as thermonuclear supernova [6, 7]. From the recently published stellar evolution models by Jones et al. [3], it becomes obvious that starting from very similar initial masses, different models lead to various final stages of stellar evolution like O-Ne white dwarfs, electron capture supernovae or core-collapse supernovae. This is mainly due to the fact that certain nuclear reactions play a decisive role in determining the fate of the star. One particular reason for this is the large density dependence of the EC processes involved, like the chain ${ }^{20} \mathrm{Ne}\left(e^{-}, v_{e}\right)^{20} \mathrm{~F}\left(e^{-}, v_{e}\right)^{20} \mathrm{O}$. They are especially relevant as they have a large impact on the temperature evolution in the center of the star. The basic mechanism of these reactions is displayed in Figure 1. Due to pairing effects, the mass parabolas for odd-A isotopes are different than those for even-A isotopes. The initial composition of the $\mathrm{O}-\mathrm{Ne}-\mathrm{Mg}$-core consists of nuclei in the mass range $A=16-27$. As the density grows and the electron Fermi energy increases, EC processes set in initially in odd-A chains where the capture threshold for stable nuclei is substantially lower (see Figure 1). However, the electron energy is not large enough to produce a second EC and the produced nucleus $\beta$-decays back to the original nucleus producing an Urca cycle [8]:

$$
\begin{aligned}
(A, Z)+e^{-} & \rightarrow(A, Z-1)+v_{e}, \\
(A, Z-1) & \rightarrow(A, Z)+e^{-}+v_{e} .
\end{aligned}
$$

In this Urca cycle, two neutrinos are emitted carrying away energy from the stellar plasma. The result of this is the very efficient Urca cooling mechanism [8].

The situation is different for an even-A chain. Due to the much larger capture threshold on stable nuclei, EC on even-even nuclei set in at higher densities than for the odd-A chain. Once the 

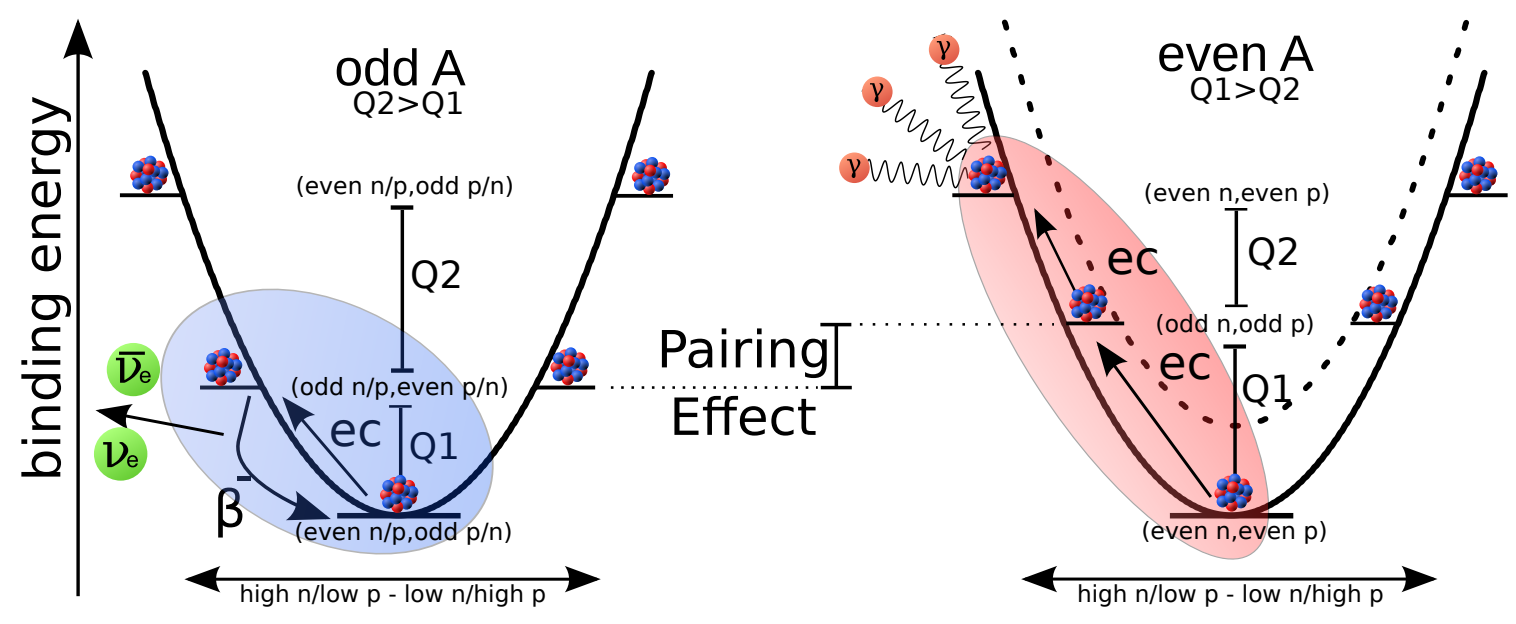

Figure 1: Schematic illustration of the mass parabola for an isobaric chain of nuclei. The left side shows odd-A nuclei, the right side even-A nuclei. The most stable nucleus is situated in the apex of the parabola. The binding energy levels get shifted due to the paring of nucleons. Under the right conditions, which can occur in stars, this leads to Urca cooling for odd-A nuclei and a heating effect for even-A nuclei.

electron Fermi energy becomes large enough to produce a capture on the even-even nucleus, it is followed immediately by a second capture on the odd-odd nucleus (see Figure 1):

$$
\begin{aligned}
(A, Z)+e^{-} & \rightarrow(A, Z-1)+v_{e}, \\
(A, Z-1)+e^{-} & \rightarrow(A, Z-2)+v_{e} .
\end{aligned}
$$

For the second step in this chain, the electron Fermi energy $E_{\mathrm{f}}$ is much higher than the capture threshold. As a consequence, the even-even nucleus is produced in a highly excited state that decays by gamma emission releasing energy that is transformed into heat in the star.

In general, the Urca cycle on odd-A chains operates for a certain time period before EC on even-A chains set in. As even-even nuclei are more abundant in the stellar core than odd-A nuclei, once EC on even-A nuclei are present, there is a net source of heating that contributes to raising the temperature in the star.

As the electrons in the star can be described as a relativistic fermi gas, $E_{f}$ follows a simple density dependence: $E_{f} \propto \rho^{1 / 3}$. Once $E_{f}$ is on the same order than the mass difference between two neighboring isobars, EC reactions occur. Reaching densities of $1-3 \times 10^{9} \mathrm{~g} \mathrm{~cm}^{-3}$, Urca cooling involving odd-A sd-shell nuclei starts to take place in the star (e.g. $\left.{ }^{23} \mathrm{Na},{ }^{25} \mathrm{Mg},{ }^{27} \mathrm{Al}\right)$. With further increasing density, also EC on even-A sd-shell nuclei start to occur and increase the temperature in the core of the star. In the beginning, ${ }^{24} \mathrm{Mg}$ is converted into ${ }^{24} \mathrm{Ne}$ and later is followed by capture on ${ }^{20} \mathrm{Ne}$ that can produce considerable amounts of ${ }^{20} \mathrm{O}$ via the reaction chain ${ }^{20} \mathrm{Ne}\left(e^{-}, v\right){ }^{20} \mathrm{~F}\left(e^{-}, v\right){ }^{20} \mathrm{O}$, before reaching Neon burning temperatures. The EC rates of ${ }^{20} \mathrm{Ne}$ have been recently reevaluated by Martínez-Pinedo et al. [9], where the authors have developed an analytic formalism that allows for a accurate description of the strong dependence in density and temperature of the stellar rates. They have also pointed out the important role that a second-forbidden transition between the ground states of ${ }^{20} \mathrm{Ne}$ and ${ }^{20} \mathrm{~F}$ plays at conditions below $0.9 \mathrm{GK}$ and densities between $1-2 \times 10^{9} \mathrm{~g} \mathrm{~cm}^{-3}$. These transitions can result in a rate that is by several orders of magnitudes larger than the previously used rate in stellar evolution calculations [10] (c.f. A. Idini's contribution to the NIC XIII 

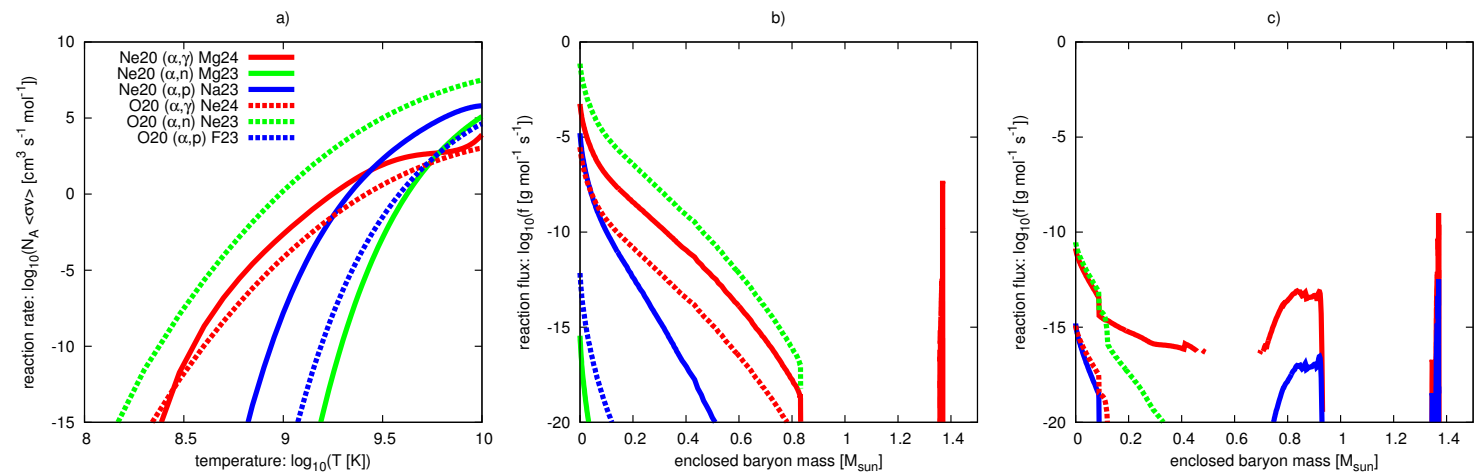

Figure 2: Panel a) shows $(\alpha,\{\gamma, \mathrm{n}, \mathrm{p}\})$-rates for ${ }^{20} \mathrm{O}$ and ${ }^{20} \mathrm{Ne}$ taken from the JINA Reaclib database [11]. Panel b) and c) show the flux of the reactions displayed in panel a) for conditions taken from profiles of two different stellar evolution models by Jones et al. prior to the neon burning phase. Panel b) corresponds to the $8.75 \mathrm{M}_{\odot}$ and panel c) to the $8.8 \mathrm{M}_{\odot}$ model.

proceedings). In the stellar evolution models of Jones et al., these conditions are present prior to the onset of the neon burning phase. Hence, we would like to point out that the inclusion of the updated EC rates can have a significant impact onto the final evolution of the star.

Due to the EC reactions mentioned above, the center of the core consists mainly of ${ }^{16} \mathrm{O}$ and ${ }^{20} \mathrm{Ne},{ }^{24} \mathrm{Ne}$ and some fraction of ${ }^{20} \mathrm{O}$ (up to $4 \%$ ). The situation is different to massive stars where no EC phase occurs before neon burning. In this case, neon burning proceeds via the following reactions:

$$
{ }^{20} \mathrm{Ne}(\gamma, \alpha){ }^{16} \mathrm{O} \quad \text { and } \quad{ }^{20} \mathrm{Ne}(\alpha, \gamma){ }^{24} \mathrm{Mg} .
$$

Due to the presence of ${ }^{20} \mathrm{O}$ however, additional reaction channels need to be taken into account:

$$
{ }^{20} \mathrm{O}(\alpha,\{\gamma, \mathrm{n}, \mathrm{p}\})\left\{{ }^{24} \mathrm{Ne},{ }^{23} \mathrm{Ne},{ }^{23} \mathrm{~F}\right\} .
$$

In panel a) of Figure 2, we compare the rates of different reaction channels for the $\alpha$-capture on ${ }^{20} \mathrm{Ne}$ and ${ }^{20} \mathrm{O}$, respectively. As can be seen, the reaction ${ }^{20} \mathrm{O}(\alpha, n){ }^{23} \mathrm{Ne}$ is by far dominating between 0.1 and $10 \mathrm{GK}$. Hence, we argue that the $\alpha$-capture on ${ }^{20} \mathrm{O}$ can be a competitive process and should be considered in future calculations. This can be seen even better in panel b) and c) where we look at the reaction fluxes for conditions of profiles of two different stellar evolution models from Jones et al. prior to the neon burning phase. For a binary rate, the flux is defined as: $f_{\mathrm{AB} \rightarrow \mathrm{X}}=\rho N_{\mathrm{A}}\langle\sigma v\rangle_{\mathrm{AB} \rightarrow \mathrm{X}} Y_{\mathrm{A}} Y_{\mathrm{B}}$, with the density $\rho$, the abundances in the incoming channels $Y_{\mathrm{A}}$ and $Y_{\mathrm{B}}$ and the reaction rate $N_{\mathrm{A}}\langle\sigma v\rangle_{\mathrm{AB} \rightarrow \mathrm{X}}$. It is obvious that for the $8.75 \mathrm{M}_{\odot}$ model where there is up to $4 \%$ of ${ }^{20} \mathrm{O}$, the reaction ${ }^{20} \mathrm{O}(\alpha, n){ }^{23} \mathrm{Ne}$ is dominant. In the case of the $8.8 \mathrm{M}_{\odot}$ model where there is only up to $0.1 \%$ of ${ }^{20} \mathrm{O}$, it is at least comparable to the ${ }^{20} \mathrm{Ne}(\alpha, \gamma){ }^{24} \mathrm{Mg}$ channel in the center of the core.

Once the star reaches temperatures in excess of $1.7 \mathrm{GK}$, the fusion of oxygen will become 

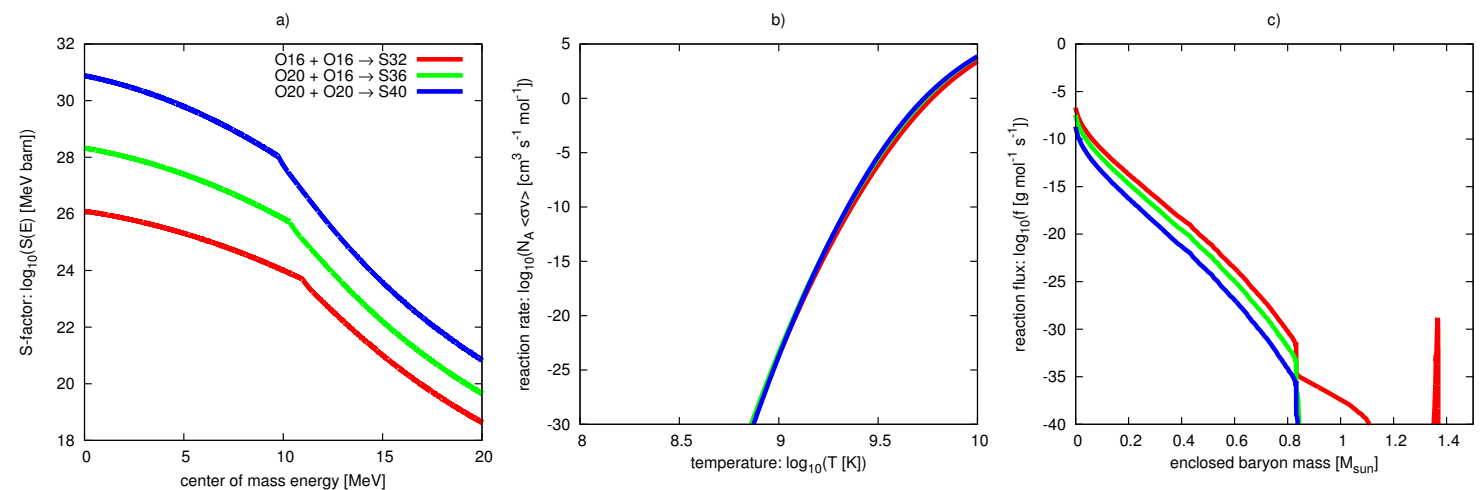

Figure 3: Panel a) shows S-factors of the different oxygen fusion channels as a function of projectile center of mass energy and panel b) shows the corresponding reaction rates. Panel c) shows the flux of each reaction for the same conditions as in panels b) and c) of Figure 2 .

important. As there is also ${ }^{20} \mathrm{O}$ available, the fusion can now proceed via three channels:

$$
\begin{gathered}
{ }^{16} \mathrm{O}+{ }^{16} \mathrm{O} \rightarrow{ }^{32} \mathrm{~S}^{*}(\mathrm{Q} \text {-value }=16.5 \mathrm{MeV}), \\
{ }^{16} \mathrm{O}+{ }^{20} \mathrm{O} \rightarrow{ }^{36} \mathrm{~S}^{*}(\mathrm{Q} \text {-value }=29.7 \mathrm{MeV}), \\
{ }^{20} \mathrm{O}+{ }^{20} \mathrm{O} \rightarrow{ }^{40} \mathrm{~S}^{*}(\mathrm{Q} \text {-value }=30.4 \mathrm{MeV}) .
\end{gathered}
$$

Due to the lack of experimental data for the fusion involving neutron-rich oxygen isotopes, we rely on a theoretical model by Yakovlev et al. [12] for the calculation of the S-factors $S(E)$ together with branching ratios that were calculated according to [13]. In this approach, the decay of the compound nucleus is described in the Hauser-Feshbach model, allowing for particle and multi-particle emission (if the excitation energy is high enough) within the framework of the MOD-SMOKER [14] and ABLA07 [15] codes. The astrophysical rate is obtained by numerical integration of the S-factor. Each individual temperature dependent rate $N_{\mathrm{A}}\langle\sigma v\rangle$ is given by:

$$
N_{\mathrm{A}}\langle\sigma v\rangle(T)=N_{\mathrm{A}}\left(\frac{8}{\pi \mu}\right)^{1 / 2}\left(k_{\mathrm{B}} T\right)^{-3 / 2} \int_{0}^{\infty} B(E) S(E) \exp \left(-E / k_{\mathrm{B}} T\right) \exp (-2 \pi \eta(E)),
$$

where $\mu$ is the effective mass and $B(E)$ is the branching ratio of each decay channel. $\eta$ is the Sommerfeld parameter: $\eta=\left(4 \pi \epsilon_{0} \hbar\right)^{-1}\left(Z_{1} Z_{2} e^{2}\right) \sqrt{\mu / 2 E}$, where $Z_{1,2}$ are the charge numbers of the reactants.

In panels a) and b) of Figure 3, it can be seen that despite the fact that the S-factors vary by several orders of magnitudes, the reaction rates are actually comparable because of the different effective masses in $\eta$. In panel c) are shown again the reaction fluxes for conditions of a profile of the $8.75 \mathrm{M}_{\odot}$ model from Jones et al.. We find that the contribution of the ${ }^{16} \mathrm{O}+{ }^{20} \mathrm{O}$-channel reaches up to $15 \%$, while the ${ }^{20} \mathrm{O}+{ }^{20} \mathrm{O}$-channel is much less important. Considering the uncertainties present in all of these reactions, it is important to perform a careful evaluation of the corresponding S-factors as they can substantially affect oxygen burning in intermediate-mass stars. In addition to that, the fusion involving ${ }^{20} \mathrm{O}$ has Q-values of $29.7 \mathrm{MeV}$ and $30.4 \mathrm{MeV}$, respectively. This does not only increase the rate of energy release during the fusion phase of oxygen by up 30\%, but it also allows for more exotic decay channels including the emission of up to 5 neutrons. 


\section{Conclusion}

We propose additional burning processes that should be considered in stellar evolution models for intermediate-mass stars as they have a strong impact onto the final evolution of these stars. In the future, we want to use these rates to continue the modeling of the final phase of stellar evolution of intermediate-mass stars, including the recently published EC rates on sd-shell nuclei, the additional rates for the neon burning as they have been pointed out in the previous section and also the fusion of the neutron-rich oxygen isotopes, including the detailed computation of the branching ratios.

\section{Acknowledgments}

H.M. is supported by the Deutsche Forschungsgemeinschaft through contract SFB 634. H. M. is member of the HGS-HIRe Graduate School. T.F. acknowledges support from the Narodowe Centrum Nauki (NCN) within the "Maestro" program under contract No. DEC- 2011/02/A/ST2/00306. G.M.P. is partly supported by the Deutsche Forschungsgemeinschaft through contract SFB 634, the Helmholtz International Center for FAIR within the framework of the LOEWE program launched by the state of Hesse, and the Helmholtz Association through the Nuclear Astrophysics Virtual Institute (VH-VI-417).

\section{References}

[1] K. Nomoto, Evolution of 8-10 solar mass stars toward electron capture supernovae. I - Formation of electron-degenerate $O+N E+M G$ cores, Astrophys. J. 277791.

[2] K. Nomoto, Evolution of 8-10 solar mass stars toward electron capture supernovae. II - Collapse of an $O+N E+M G$ core, Astrophys. J. 322206.

[3] S. Jones, et al., Advanced Burning Stages and Fate of 8-10 M $\odot$ Stars, Astrophys. J. 772150.

[4] K. Takahashi, T. Yoshida, H. Umeda, Evolution of Progenitors for Electron Capture Supernovae, Astrophys. J. 77128.

[5] Z. G. Jennings, et al., Supernova Remnant Progenitor Masses in M31, Astrophys. J. 76126.

[6] J. Isern, R. Canal, J. Labay, The outcome of explosive ignition of ONeMg cores - Supernovae, neutron stars, or 'iron' white dwarfs?, ApJ. 372 L83.

[7] J. Gutierrez, E. Garcia-Berro, I. Iben, Jr., J. Isern, J. Labay, R. Canal, The Final Evolution of ONeMg Electron-Degenerate Cores, ApJ. 459701.

[8] S. Tsuruta, A. G. W. Cameron, URCA Shells in Dense Stellar Interiors, Ap\&SS7 374.

[9] G. Martínez-Pinedo, Y. H. Lam, K. Langanke, R. G. T. Zegers, C. Sullivan, Astrophysical weak-interaction rates for selected $A=20$ and $A=24$ nuclei, Phys. Rev. C 89045806.

[10] M. Takahara, et al., Microscopic calculation of the rates of electron captures which induce the collapse of $\mathrm{O}+\mathrm{Ne}+\mathrm{Mg}$ cores, Nucl. Phys. A 504167.

[11] R. H. Cyburt, et al., The JINA REACLIB Database: Its Recent Updates and Impact on Type-I X-ray Bursts, Astrophys. J. Suppl. 189240.

[12] D. G. Yakovlev, M. Beard, L. R. Gasques, M. Wiescher, Simple analytic model for astrophysical S factors, Phys. Rev. C 82044609. 
[13] L. Huther, Nucleosynthesis in neutrino-driven winds, Ph.D. thesis, TU Darmstadt, Darmstadt, Germany (2014), URL http://tuprints.ulb.tu-darmstadt .de/3766/.

[14] H. P. Loens, Microscopic radiative strength functions and fission barriers for r-process nucleosynthesis, Ph.D. thesis, TU Darmstadt, Darmstadt, Germany (2011), URL http://tuprints.ulb.tu-darmstadt.de/2695/.

[15] A. Kelic, M. Valentina Ricciardi, K.-H. Schmidt, ABLA07 - towards a complete description of the decay channels of a nuclear system from spontaneous fission to multifragmentation, ArXiv e-prints [0906.4193]. 